\title{
Uterine Liposarcoma in a German Shephard Dog
}

\author{
Vullo Cecilia $^{1}$, Russo Marco ${ }^{2}$, Mariotti Francesca ${ }^{1}$, England C. W. Gary ${ }^{3}$, Catone Giuseppe ${ }^{1}$ \\ ${ }^{1}$ School of Veterinary Medicine, Camerino University, Camerino, Italy \\ ${ }^{2}$ Veterinary Science Department, Naples University, Naples, Italy \\ ${ }^{3}$ School of Veterinary Medicine and Science, University of Nottingham, Nottingham, UK \\ Email: francesca.mariotti@unicam.i
}

Received February 13, 2013; revised March 23, 2013; accepted April 23, 2013

Copyright (C) 2013 Vullo Cecilia et al. This is an open access article distributed under the Creative Commons Attribution License, which permits unrestricted use, distribution, and reproduction in any medium, provided the original work is properly cited.

\begin{abstract}
Uterine tumours in the bitch are considered rare. The most common are leiomyomas representing more than the $90 \%$ of all uterine canine tumours. Very few cases of uterine malignant tumours have been described in the veterinary literature and uterine liposarcoma had never discovered. Also in human medicine lipomatous tumours, whether benign or malignant, are very rare in the uterus. These tumours may arise from the misplacement of an embryonic progenitor cell, metaplasia of mature mesenchymal tissues of other types, perivascular adipocytes or from traumatic displacement of adipocytes. The authors describe a case of unilateral liposarcoma in the uterus of a German Shepherd dog and its radiographic, ultrasonographic evaluation and the histopathological appearance.
\end{abstract}

Keywords: Uterin Tumors; Liposarcoma; Immunohistochemistry; Dog

\section{Introduction}

Liposarcomas are rare malignant tumours of white adipose cell lipoblasts [1]. They are considered a soft tissue neoplasm because of their mesenchymal or connective tissue cell origin. They often arise from the skin and subcutis [2]. Most reports indicate that liposarcomas are unlikely to metastasize; however, a few published reports suggest that liposarcomas are aggressive, locally invasive, and commonly metastasize to the lungs, liver, and bone [3].

Uterine tumours in the bitch are considered rare, accounting for only $0.4 \%$ of all canine tumours, and between $1 \%$ to $19 \%$ of all the female genital tract tumours [3-5]. The most common are leiomyomas representing more than the $90 \%$ of all uterine canine tumours [1]. It is thought that steroid hormones play a role in the pathogenesis of leiomyomas [2]. However, malignant tumour diseases in dogs are extremely rare with very few cases described in the veterinary literature [6].

Tumours of the canine genital system are usually detected during necroscopy or ovariohysterectomy $[7,8]$. Clinical signs are dependant on the size and location of the tumour. Sometimes there are no consistent clinical signs to indicate cancer involvement in the uterus other than the presence of an abdominal enlargement that can be revealed by a physical examination [3].

In this study, clinicopathological and immunohisto- chemical findings of canine unilateral liposarcoma were described.

\section{Case Report}

A 10 years old German Shepard bitch was brought to the Veterinary Teaching Hospital of the University of Camerino for history of intermittent inappetence. On physical examination a localised left caudodorsal bulging was noted and on palpation an abdominal mass discovered. The mass appear to be quite firm and extended from the third to seventh intervertebral space. Abdomen radiographic examination in a right lateral view was performed. Radiographic finding was indicative of a large and not well-defined mass, with mixed soft tissue opacity and irregular marginated (Figure 1).

Realtime B mode ultrasonography equipment (Esaote, Mylab $30 \mathrm{Vet}$ ) was used with $5-8 \mathrm{MHz}$ microconvex probe in order to perform an abdominal examination. Dorsal to the urinary bladder, just cranial to the uterine bifurcation a well marginated mass was noted. The mass appeared to be eterogeneus in ecotexture for the presence of multiple cyst like lesion mixed with areas of hypoecogenicity (Figure 2). On the colour Doppler examination the mass showed no internal colour flow and a very feeble colour contour outside the capsule. All the other organs were within normal limits.

In order to see if thoracic metastasis were present 


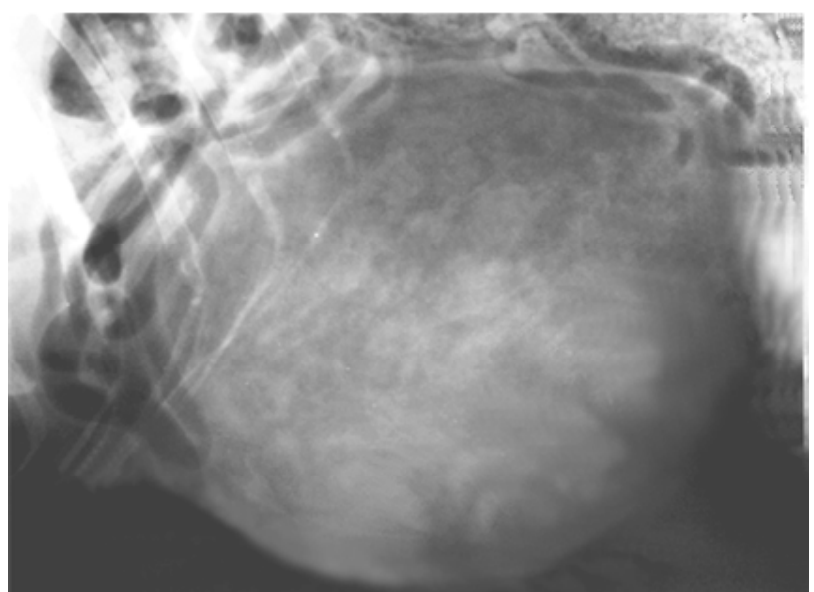

Figure 1. Right lateral abdominal radiograph. There is a large and not well-defined mass, with mixed soft tissue opacity and irregular marginated.

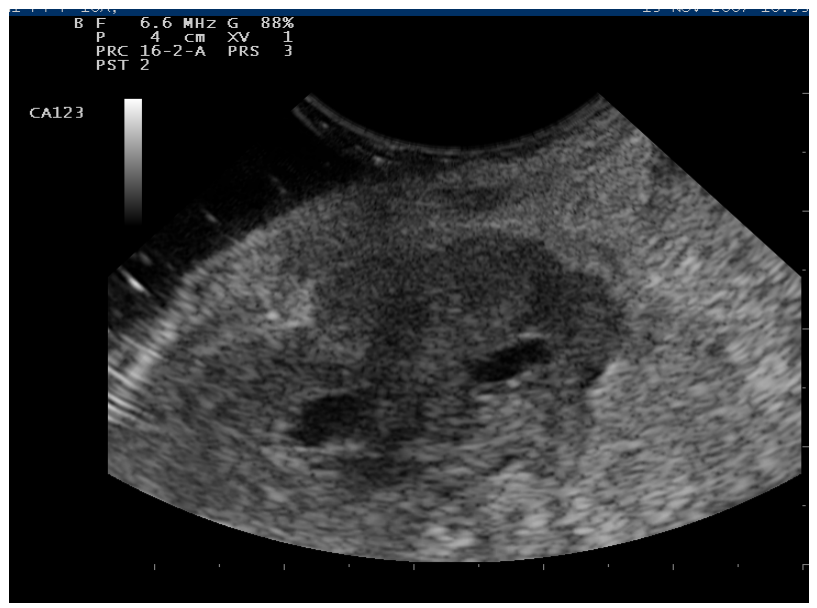

Figure 2. Ultrasonographic image of the abdominal mass. It appeared to be eterogeneus in ecotexture for the presence of multiple cyst like lesion mixed with areas of hypoecogenicity.

right-lateral thoracic radiograph was done and no abnormality was detected.

Prior the surgery a complete serum haematology and biochemistry were performed.

The dog was premedicated with $5 \mu \mathrm{g} / \mathrm{kg}$ intramuscular medetomidine and $0.3 \mathrm{mg} / \mathrm{kg}$ intramuscular tramadol. Anaesthesia was induced with $2 \mathrm{mg} / \mathrm{kg}$ intravenous propofol and general anaesthesia was maintained with isoflurane in oxygen.

An exploratory laparotomy was performed via a midline incision and an irregular-shaped, firm mass, $21 \times 17$ $\times 12 \mathrm{~cm}$ in size, was identified in the uterine left horn.

Ovariohysterectomy was performed to remove the mass (Figure 3). Recovery of the bitch was rapid and uneventful. Samples of the mass were fixed in $10 \%$ buffered formalin and subjected to routine histopathological evaluation.

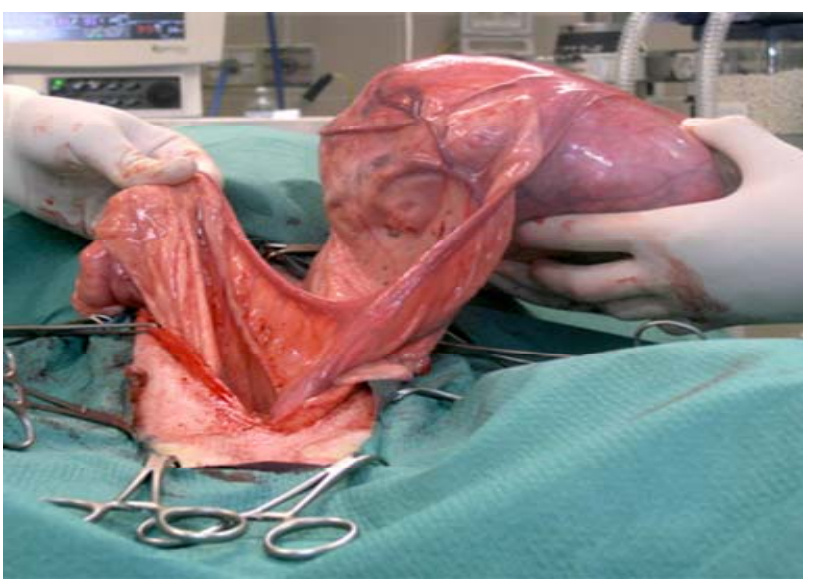

Figure 3. Intraoperative image. Note the mass in the left uterine horn.

With E-E staining, the neoplastic tissue appeared costituited by relatively mature adipocytes with a single clear fat vacuole and a peripheral oval nucleus or with cytoplasm containig variably sized lipid droplets and round nucleus (Figure 4). The size of neoplastic cells were variable and in a proportion of cells, atypical and hyperchromatic nuclei were found. Admixed with these cells are scattered bizarre, often multinucleate, stromal cells and variably prominent fibrous septa containing hyperchromatic spindle cells and occasional bizarre cells.

For immunohistochemical investigations, $3 \mu \mathrm{m}$-sections were cut from each specimen and labelled for the presence of Vimentin, pancytokeratin (CK), S-100 and Ki-67 antigens (Dako Corporation, Carpinteria, CA, USA). Immunohistochemical reaction was detected using an avidinbiotin-peroxidase method according to manufacturer's instructions (Vector Laboratories, Inc., Burlingame, CA, USA). A solution of $25 \mathrm{mg}$ of 3-3'diaminobenzidine (DAB) was used as the chromogen while nuclear counterstaining was performed with Mayer's haematoxylin.

Immunohistochemically, the neoplastic cells were vimentin-positive and CK-negative (Figures 5 and 6).

The proportion of labelled cells with anti-Ki-67 antibody varied from $26 \%$ to almost $75 \%$ and the intensity varied from moderate to intense. An occasional positivity to S-100 antigen was found (Figure 7).

\section{Discussion}

Liposarcoma, malignant counterpart to the lipoma, is rare in domestic animals, probably most common in the canine species. It is a typical tumour of the skin and soft tissues. There is no sex predisposition but the incidence increases with age [9].

Very few cases of uterine malignant tumours have been described in the veterinary literature [6,10-13] and uterine liposarcoma was never discovered.

Being the most massive overgrowth was not possible 


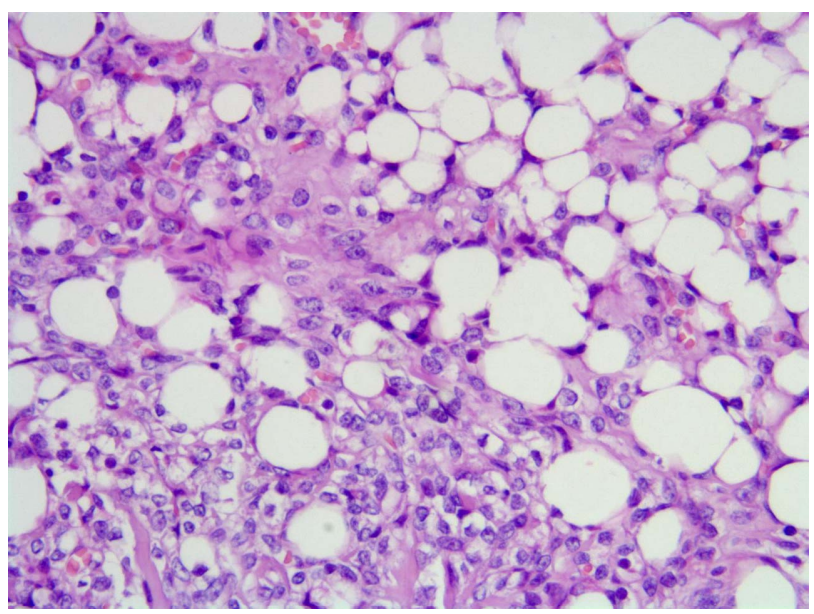

Figure 4. Neoplastic cells with a single clear fat vacuole and a peripheral oval nucleus EE, $20 \times$ ).

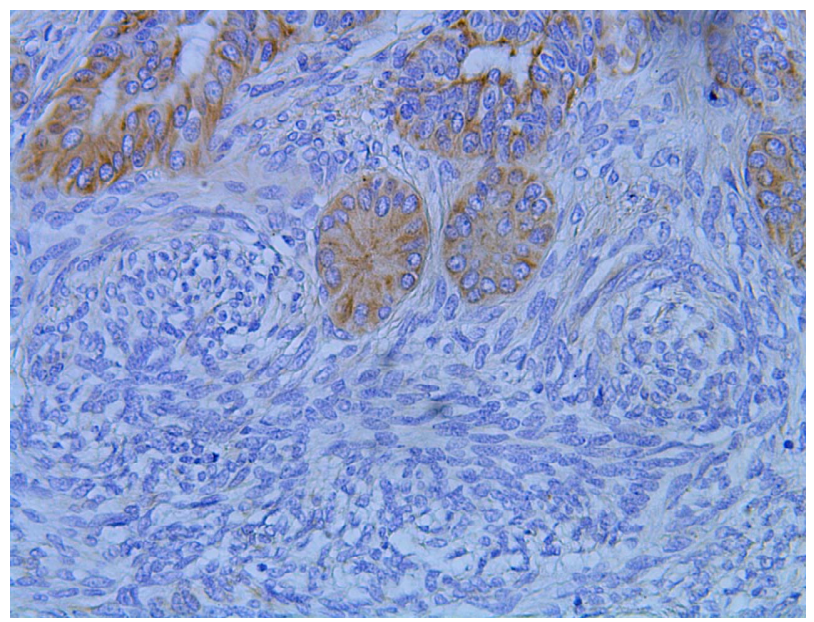

Figure 5. Neoplastic spindle cells CK-negative. Immunostain for panCK $20 \times$.

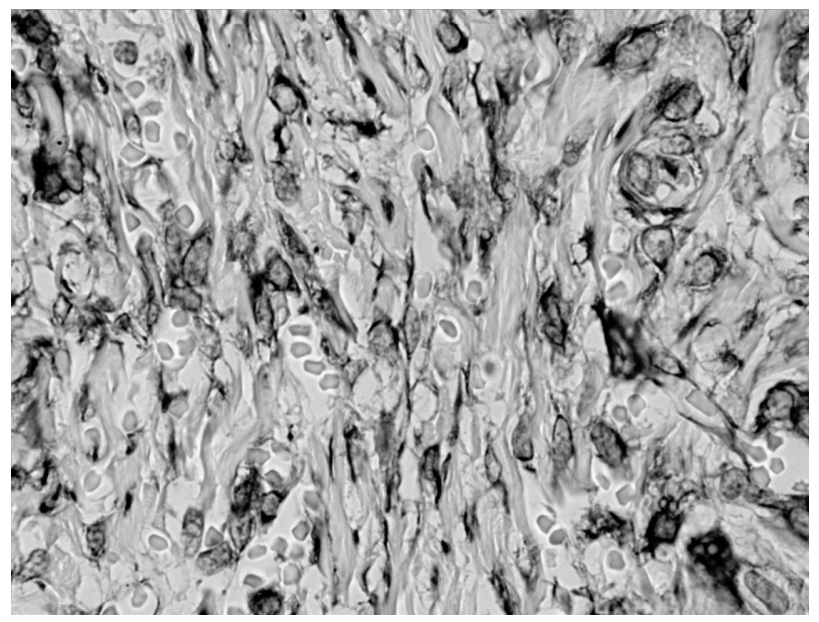

Figure 6. The neoplastic cells were vimentin-positive. Immunostain for Vimentin, $40 \times$.

to properly display the genital tract, making it uncom-

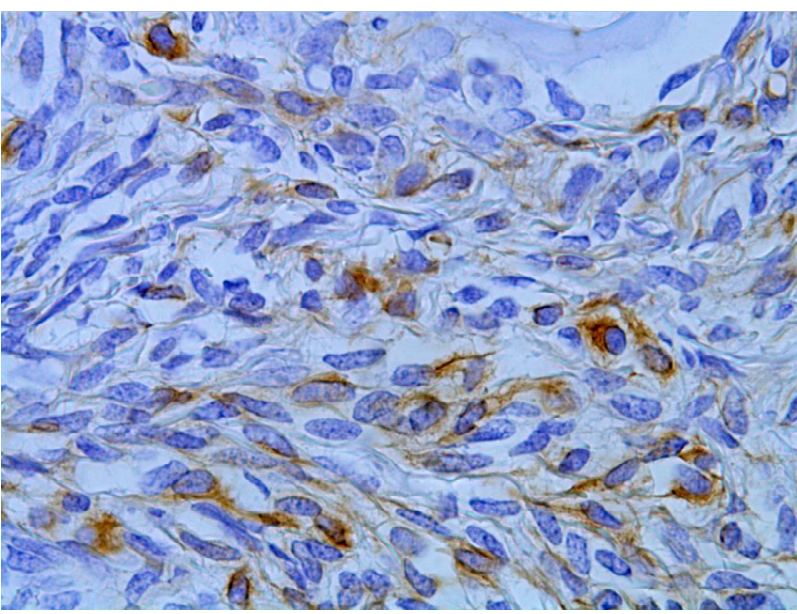

Figure 7. An occasional positivity to S-100 antigen was found. Immunostain for $\mathrm{S}-100,40 \times$.

fortable identifying the exact origin of it. The exploratory laparotomy was performed to identify the relevance of the uterine mass.

Excision of the mass associated with ovariohysterectomy has proven therapy of choice as a decisive and uncomplicated. Given the size of the mass, we evaluated the uterine liposarcoma of the dog is likely to slow tumour growth and with little tendency to metastasis.

To the author's knowledge, this is the first case of a uterine liposarcoma in a bitch.

\section{REFERENCES}

[1] K. V. F. Jubb, P. C. Kennedy and N. C. Palmer, "Female Genital Tract," Pathology of Domestic Animals, 5th Edition, Elsevier Saunders, 2007.

[2] D. A. Fiorito, "Hyperestrogenism in Bitches," Compendium on Continuing Education for the Practicing Veterinarian, Vol. 14, 1992, pp. 727-729.

[3] W. B. Morrison, "Cancer of the Reproductive Tract," Cancer in Dogs and Cats: Medical and Surgical Management, 2nd Edition, TNM Publishing, 2002.

[4] S. D. Johnston, M. V. Root Kustritz and P. N. Olson, "Canine and Feline Theriogenology," Saunders, Philadelphia, 2001.

[5] M. D. McGavin and J. F. Zachary, "Female Reproductive System," Pathologic Basis of Veterinary Disease, 4th Edition, Mosby Elsevie, 2007.

[6] F. J. Pena, J. A. Gines, J. Duque, R. Martinez-Pe're, L. Madejo, I. N. Martinez, J. M. Moran and S. FernandezGarcia, "Endometrial Adenocarcinoma and Mucometra in a 6 Years Old Alaska Malamute Dog," Reproduction in Domestic Animals, Vol. 41, No. 2, 2006, pp. 189-190. doi:10.1111/j.1439-0531.2006.00645.x

[7] K. Eker, M. R. Salmanoglu and S. A. Vural, "Unilateral Leiomyoma in the Mesosalpinx of a Dog," Journal of the American Animal Hospital Association, Vol. 42, 2006, pp. 392-394. 
[8] D. J. Meuten, "Tumors of the Genital System. Tumors of the Skin and Soft Tissues," Tumors in Domestic Animals, 4th Edition, Blackwell Publishing, 2002. doi: $10.1002 / 9780470376928$

[9] W. B. Morrison, "Cancer of Reproductive Tract," Cancer in Dogs and Cats, TNM Publishing, 2002.

[10] C. E. Payne-Johnson, D. F. Kelly and P. T. Davies, "Endometrial Carcinoma in a Young Dog," Journal of Comparative Pathology, Vol. 96, No. 4, 1986, pp. 463-467. doi:10.1016/0021-9975(86)90042-3

[11] Y. Murakami, K. Uchida, R. Yamaguchi and S. Tateyama, "Diffuse Bilateral Hemangiosarcoma of the Uterus of a
Dog," Journal of Veterinary Medical Science, Vol. 63, 2001, pp. 191-193. doi:10.1292/jvms.63.191

[12] T. A. Cave, R. Hine, F. Howie, H. Thompson and D. J. Arglye, "Uterine Carcinoma in a 10-Old-Month Golden Retriever," Journal of Small Animal Practice, Vol. 43, No. 3, 2002, pp. 133-135. doi:10.1111/j.1748-5827.2002.tb00044.X

[13] Y. K. Choi, J. Y. Lee, D. Y. Kim, J. I. Park, S. W. Jeong, H. M. Park and C. Park, "Uterine Extramedullary Plasmacytoma in a Dog," Veterinary Record, Vol. 22, 2004, pp. 699-700. doi:10.1136/vr.154.22.699 